\title{
Empirical correction of low Sun angle images in steeply sloping terrain: a slope-matching technique
}

\author{
J. NICHOL*, LAW KIN HANG and WONG MAN SING \\ Department of Land Surveying and Geo-Informatics, The Hong Kong Polytechnic \\ University, Hung Hom, Kowloon, Hong Kong
}

(Received 4 May 2005; in final form 8 August 2005)

\begin{abstract}
A technique based on slope matching is presented, which corrects the topographic effect on images with very low illumination due to very steep terrain, very low Sun angle, or both. The technique is a modification of Civco's (1989) two-stage normalization correction. The modified correction arose from the failure of existing correction methods to eliminate dark shadows on wintertime IKONOS images, in the steeply sloping terrain of our study area. The shadows, which were particularly severe in the near-infrared (NIR) band preclude accurate habitat classification due to the importance of this band on IKONOS multispectral images: the remaining three bands being highly correlated. Since the objective of topographic correction is to equalize the radiance between shady and sunny slopes, the slope-matching technique normalizes the radiance values to the mean of the sunny slope, rather than to the overall mean, as in the two-stage normalization correction of Civco (1989). This enables a more appropriate correction factor to be computed, suitable for the wide range of values encountered for the incident angle of illumination. The slope-matching correction was able to reduce intra-class variance significantly more than the two-stage normalization correction, as well as increase classification accuracy by $7 \%$.
\end{abstract}

\section{Introduction}

Due to its steeply sloping terrain, over $40 \%$ of the territory of Hong Kong, an area of approximately $450 \mathrm{~km}^{2}$, has been designated as country parks. Effective management of these areas requires detailed and accurate ecological maps. Although several mapping projects have been undertaken, positional and class accuracy have been low due to problems of orthorectification of aerial photographs. Although high quality aerial photography is available, this suffers from geometric, off-nadir effects of radial displacement compounded by relief displacement, thus requiring orthorectification of each photo individually. Detailed ecological maps from a single mapping base are potentially available from IKONOS multispectral images. However, as with air photos, a further problem in the interpretation of cover types is the differential Sun illumination effect on opposing slopes. The relative relief of Hong Kong's country parks is extreme, with elevations rising from sea level to over $900 \mathrm{~m}$, within a $4 \mathrm{~km}$ planimetric distance, and average slopes approximating $23^{\circ}$.

*Corresponding author. Email: 1sjanet@polyu.edu.hk 
The illumination differences are compounded by the preference for wintertime imagery for ecological mapping, due to greater contrast between the main structural cover types, broadleaf evergreen forest, shrub and grassland. Sun elevation angles are typically $38-42^{\circ}$ at mid-morning. While forest maintains high photosynthesis during the winter dry season, grassland, mainly occupying summits, dies back and exhibits much lower near-infrared (NIR) reflectance. Shrubland areas also become semi-senescent during the winter dry season due to a less established root system, with NIR reflectance mid-way between that of grassland and forest. Furthermore, high Sun angle, summertime imagery is rarely available in Hong Kong due to cloud cover. In radiometric terms, a prerequisite for ecological mapping using automated techniques is a flat surface representation of the original image. Since the only model able to provide such an image (Shepherd and Dymond 2003) requires detailed atmospheric data, this analysis demonstrates an empirical technique based on slope matching, which is effective for images with low illumination values. It is an adaptation of Civco's (1989) two-stage normalization method.

\section{Background to the present investigation}

Some existing methods for topographic correction were tested for a summertime IKONOS image of the study area, a $25 \mathrm{~km}^{2}$ area of Shing Mun country park, Hong Kong. These included the Cosine method (Smith et al. 1980, Teillet et al. 1982), the Minnaert correction (Minnaert 1941, Smith et al. 1980, Holben and Justice 1980, Teillet et al. 1982) and the two-stage normalization method (Civco 1989). The best result was obtained from Civco's two-stage normalization correction. Specifically, the Cosine method over-corrected for shady slopes due to the relatively higher contribution of diffuse reflectance to the overall image radiance, resulting in higher values on shady, than on sunny slopes. This problem has also been pointed out by others (Holben and Justice 1980, Smith et al. 1980, Teillet et al. 1982, Meyer et al. 1993). The Minnaert method attempts to correct for the non-Lambertian behaviour of different materials, therefore an a priori assumption of cover types is required.

The two-stage normalization method gave the most satisfactory result in terms of visual effect, and was the only technique tested that reduced the variance of the whole image as well as within classes (Law 2005). This method is based on the creation of a shaded relief model from a digital elevation model (DEM), where illumination values are computed from the Sun elevation and azimuth angles at the image time. The computed illumination values ranging from -1 to +1 are scaled to a range of $0-255$. The image $\mathrm{DN}$ values are then normalized using the illumination model in equation (1).

$$
\delta \mathrm{DN}_{\lambda i j}=\mathrm{DN}_{\lambda i j}+\left(\mathrm{DN}_{\lambda i j} \times \frac{\left(\mu_{k}-X_{i j}\right)}{\mu_{k}}\right)
$$

where $\delta \mathrm{DN}_{\lambda i j}$ is the normalized $\mathrm{DN}$ data for pixel $i j$ in band $\lambda ; \mathrm{DN}_{\lambda i j}$ is the raw $\mathrm{DN}$ data for pixel $i j$ in band $\lambda ; \mu_{k}$ is the mean value of the scaled (0-255) illumination model for the main cover type; and $X_{i j}$ is the scaled (0-255) illumination value for pixel $i j$ for the main cover type.

While Civco (1989) observed that this first stage normalization procedure maintains the mean spectral response of the image data while reducing the variance, he noted that the topographic effect was not completely removed and shady slopes remained dark relative to sunny slopes. In order to reduce the topographic effect 
further a second stage normalization involving an empirically determined calibration coefficient $(C)$ was introduced. The coefficient $C$ is computed from the results of the first stage correction. Spectral responses from samples of the main cover type on slopes facing to and away from the Sun are obtained by creation of training areas. The correction coefficient is determined using equation (2).

$$
C_{\lambda}=\left(\mu_{\lambda}-N_{\lambda}\right) /\left(\left(\mu_{\lambda}-N_{\lambda}\right)-\left(\mu_{\lambda}-N_{\lambda}^{\prime}\right)\right)+\left(\mu_{\lambda}-S_{\lambda}\right) /\left(\left(\mu_{\lambda}-S_{\lambda}\right)-\left(\mu_{\lambda}-S_{\lambda}^{\prime}\right)\right)
$$

where $C_{\lambda}$ is the correction coefficient for band $(\lambda) ; \mu_{\lambda}$ is the overall mean DN value for the main cover type; $N_{\lambda}$ is the mean DN value on slopes facing away from the Sun in uncalibrated data for the main cover type; $N_{\lambda}^{\prime}$ is the mean DN value on slopes facing away from the Sun in uncalibrated data after first stage normalization for the main cover type; $S_{\lambda}$ is the mean DN value on slopes facing to the Sun in uncalibrated data for the main cover type; and $S_{\lambda}^{\prime}$ is the mean DN value on slopes facing to the Sun after first stage normalization for the main cover type.

The second stage correction is then applied to the original image data:

$$
\delta \mathrm{DN}_{\lambda i j}=\mathrm{DN}_{\lambda i j}+\left(\mathrm{DN}_{\lambda i j} \times \frac{\left(\mu_{k}-X_{i j}\right)}{\mu_{k}}\right) \times C_{\lambda}
$$

It is likely that the greater accuracy of this two-stage normalization technique over other methods is due to its being both wavelength-dependent and scene-dependent, and thus it accounts simultaneously for the geometric effects of illumination and the radiometric effects of atmospheric scattering. Civco (1989) achieved between 61\% and $69 \%$ reduction in the variance introduced by the topographic effect on Landsat images for a forest cover type. However, he did not test the method for image classification, or for increasing class separability. A summertime IKONOS image of the present study area, with a Sun elevation angle of $61^{\circ}$ was corrected adequately using this method (Law 2005). However, after correcting a wintertime image with Sun elevation angle of $40^{\circ}$, the result was not deemed adequate to proceed to the stage of image classification, since dark shadows, covering $15 \%$ of the image area, remained on the steep, north-west facing slopes. This was attributed to the extreme characteristics of the low Sun elevation angle imagery $\left(40^{\circ}\right.$ on 24 November 2004) and ruggedness of the study area combined. Thus, values of the illumination model were approximately below 40 . The shadows were particularly severe in the NIR band. In these areas, mean DN values of 200, were far below the overall mean values of approximately 440. The NIR band in IKONOS is essential for any vegetationbased classification scheme since the remaining three visible bands are highly correlated. Therefore the two-stage normalization method was inadequate for our data.

\section{The adapted two-stage normalization correction}

The magnitude of the second stage Civco correction is small for very dark pixels because the extent of correction cannot be greater that the original $\mathrm{DN}$ value. We perceive the failure of Civco's method to be due to the extremely large range of DN values in the NIR region, for the main cover type (forest). This makes the mean value, which is used for computing $C$, and to which the whole image is normalized, inadequate for extreme pixel values. Therefore, since the objective of the correction is to equalize the radiance between shady and sunny slopes, it is more appropriate to 
make the extent of the correction equal to the range of radiance values $\left(\mathrm{DN}_{\max }-\mathrm{DN}_{\min }\right)$, i.e. the greatest difference between the sunny and shady slopes. Therefore, our improved slope-matching correction normalizes the radiance values to the mean of the sunny slope rather than to the overall mean. Accordingly, the first stage of the two-stage normalization equation is adapted as follows.

\subsection{The first stage}

$$
\delta \mathrm{DN}_{\lambda i j}=\mathrm{DN}_{\lambda i j}+\left(\mathrm{DN}_{\lambda \text { max }}-\mathrm{DN}_{\lambda \text { min }}\right) \times\left(\frac{\mu_{k}-X_{i j}}{\mu_{k}}\right)
$$

where notation is as for equation (1), except that $\mu_{k}$ is the mean value of the scaled illumination model for the main cover type on sunny slopes rather than the overall mean; $\mathrm{DN}_{\lambda \max }$ is the maximum $\mathrm{DN}$ value for main cover type; and $\mathrm{DN}_{\lambda \min }$ is the minimum DN value for main cover type

Thus, the DN values are normalized to the illumination level of pixels on the sunny slope rather than the mean illumination value for the entire image.

\subsection{The second stage}

The calculation of the correction coefficient (equation (5)) is also different from that of Civco's (equation (2)). Our adapted $C$ determines the difference between sunny and shady slopes, respectively, as a ratio of the mean cover type of the sunny slope, and thus normalizes the image to the mean cover type of the sunny slope.

$$
C_{\lambda}=\frac{S^{\prime}-N}{N^{\prime}-N}
$$

where $S^{\prime}$ is the mean on sunny slopes after first stage normalization; $N$ is the mean on shady slopes on raw image; and $N^{\prime}$ is the mean on shady slopes after first stage normalization

As with Civco's correction, the input to stage 2 is the raw uncorrected data, but equation (6) is used instead of equation (3).

$$
\delta \mathrm{DN}_{\lambda i j}=\mathrm{DN}_{\lambda i j}+\left(\mathrm{DN}_{\lambda \text { max }}-\mathrm{DN}_{\lambda \text { min }}\right) \times\left(\frac{\mu_{k}-X_{i j}}{\mu_{k}}\right) \times C
$$

\section{Results}

Figure 1 shows that topographic variability in the original image (figure 1(a)) was incompletely reduced by two-stage normalization (figure 1(b)). Areas of low illumination are still dark and a three-dimensional relief effect is still evident. However, following slope matching, a flat surface representation of the original image is produced, the three-dimensional relief effect is lost and the scene looks flat (figure $1(c)$ ). This image would be expected to show less variability in spectral signatures for the habitat classes, and thus greater separability between classes. This is shown in figure $1(c)$ and $(d)$. Some remaining dark areas on the corrected image correspond to pure stands of the deciduous tree, Melaleuca quinquinervia, currently leafless, which were previously inseparable from evergreen vegetation in shady areas (figure $1(a)$ and $(b)$ ). 


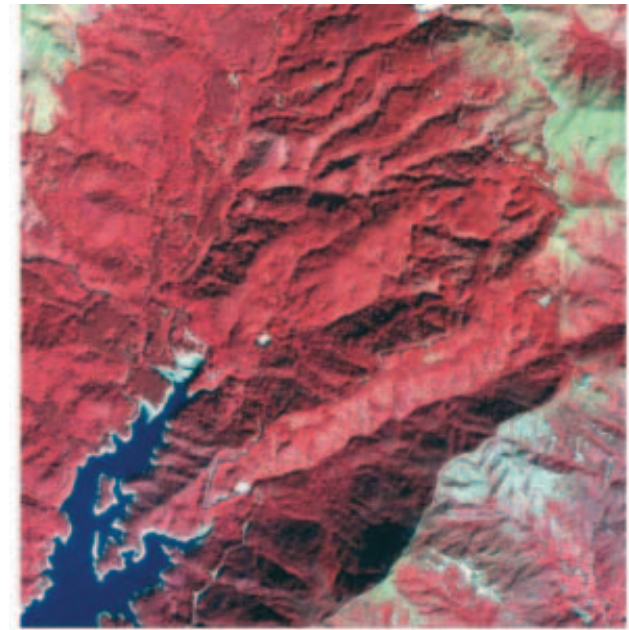

(a)

$2 \mathrm{~km}$

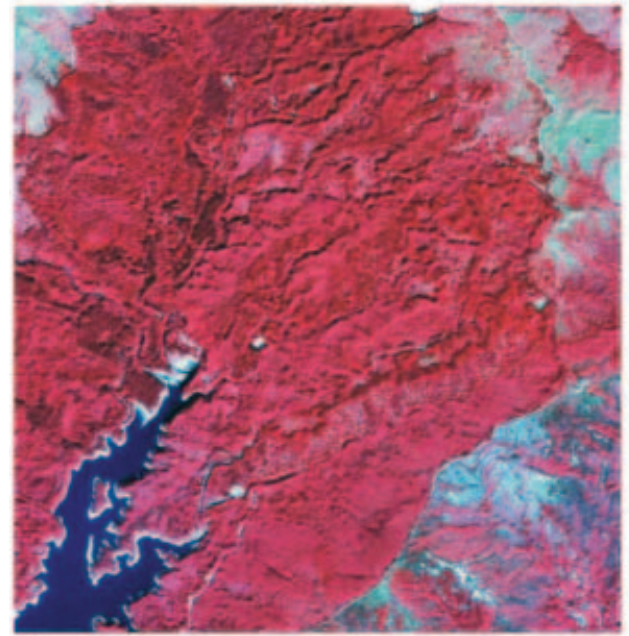

(c)

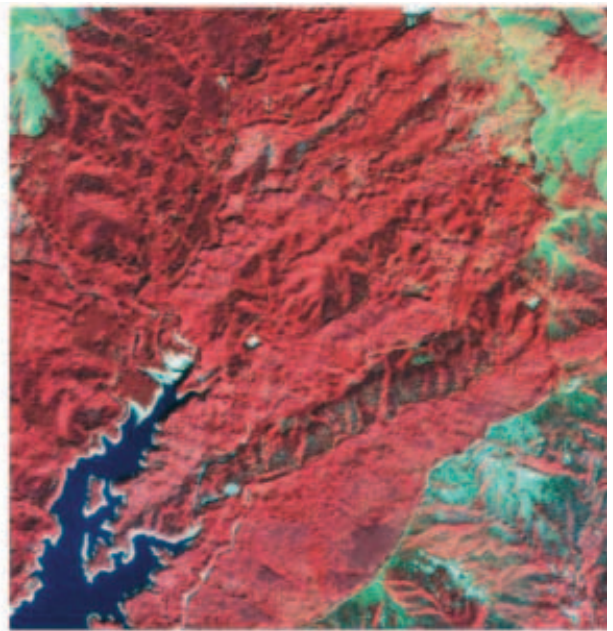

(b)

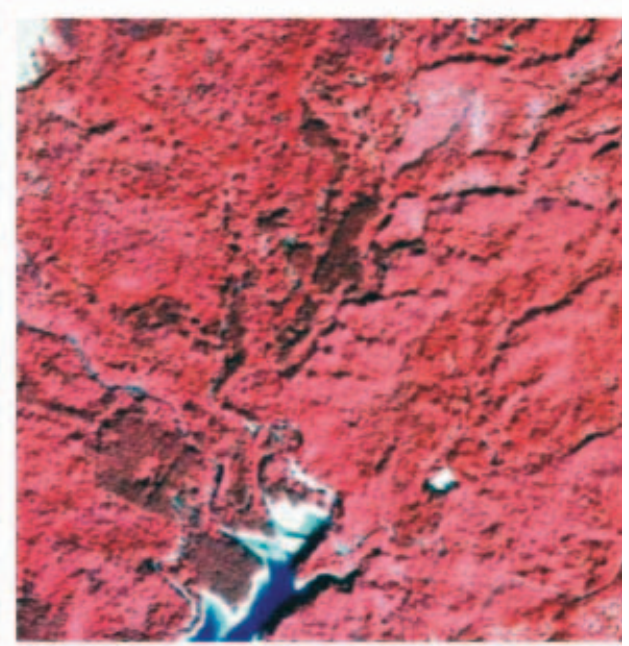

(d)

Figure 1. False colour image extract of study area showing $(a)$ the original image, $(b)$ the result of two-stage normalization, $(c)$ the result of slope matching, and $(d)$ enlargement of left centre of $(c)$, showing deciduous plantations of Melaleuca quinquinervia (to north and west of the reservoir, dark toned due to leaf loss), which are now spectrally separable from the other evergreen vegetation types.

Table 1 shows the means and standard deviations of the six habitat classes. While the two-stage normalization method is able to lower the class variance only slightly for some classes, the variance of other classes has actually increased. However the slope matching method reduces the variances more, except for the water and plantation classes (because neither class in the original image was distributed on opposing slopes, the water surfaces being flat, and plantations being located mainly on sunny, south-east slopes to replace forest lost to fire).

Table 2 shows the users and producers accuracies computed from a maximum likelihood classification for six habitat types, referenced against 335 GPS-located 
Table 1. Mean and SD (DN values) for NIR band for six habitat classes.

\begin{tabular}{|c|c|c|c|c|c|c|c|c|}
\hline \multicolumn{3}{|c|}{ Original image } & \multicolumn{3}{|c|}{ Two-stage normalisation } & \multicolumn{3}{|c|}{ Slope matching } \\
\hline Class & Mean & SD & Class & Mean & SD & Class & Mean & SD \\
\hline Water & 122 & 5 & Water & 114 & 4 & Water & 171 & 5 \\
\hline Soil & 548 & 110 & Soil & 390 & 117 & Soil & 547 & 107 \\
\hline Shrubland & 520 & 55 & Shrubland & 362 & 73 & Shrubland & 513 & 46 \\
\hline Plantation & 350 & 42 & Plantation & 294 & 40 & Plantation & 377 & 42 \\
\hline Grassland & 384 & 57 & Grassland & 306 & 55 & Grassland & 407 & 33 \\
\hline Forest & 395 & 123 & Forest & 409 & 116 & Forest & 487 & 91 \\
\hline
\end{tabular}

Table 2. User's and producer's accuracy (\%) from slope-matching correction. Numbers in parentheses refer to two-stage normalization.

\begin{tabular}{lcc}
\hline Class & Producer's accuracy & User's accuracy \\
\hline Grassland & $69(62)$ & $76(70)$ \\
Shrubland & $68(68)$ & $44(40)$ \\
Bare soil & $77(40)$ & $54(26)$ \\
Forest & $53(55)$ & $44(42)$ \\
Plantation & $54(43)$ & $78(75)$ \\
Overall accuracy: 62 (55) & & \\
\hline
\end{tabular}

field points. The overall accuracy increased by 7\% compared with the two-stage normalization correction, and all classes show increased accuracy. Specifically, after slope matching, the producer's accuracy for grassland increased from 0.62 to 0.69 , with fewer grassland pixels misclassified as soil and plantation. Additionally, the producer's accuracy for soil increased from 0.40 to 0.77 due to fewer grass and plantation pixels misclassified as soil.

\section{Discussion}

Extremely dark shadows on low Sun angle images in steeply sloping terrain are accentuated in the NIR region. On the other hand, pixels representing vegetation are unusually bright in this region, giving the NIR band a very high variance. Correcting both the high and low values on opposing slopes to the mean value using a constant, as in Civco's second stage, is unable to eliminate dark shadow effects in the NIR region. Civco (1989, table 4) found that the variance in some Landsat bands actually increased after applying the two-stage normalization correction. The same increase is true of our IKONOS data, using this correction (table 1). This situation is referred to by Teillet et al. 1982) when discussing cosine corrections of a similar form to the calculation of the illumination model input to the first stage of the two-stage normalization correction. These normally fail due to the wide range of values encountered for the incident angle of illumination, which require large correction factors, unlike those in the middle of the range. Since it is important to retain the relationship between all the bands in the multispectral image, our slope-matching technique is applied to all image bands.

The overall accuracy achieved following slope matching is still too low. However, as with most ecological classifications much of the remaining spectral confusion is due to the fuzziness of boundaries on the ground. In the study area, plantations have 
become mixed with native forest over time, and natural succession in grassland involves invasion by shrub. Since the slope-matching correction gives an acceptable result in removing spectral variation due to topography, further processing using textural and contextual classifiers can now be carried out.

\section{Acknowledgement}

Research grant PolyU2070/02E from the CERG of the government of Hong Kong supported this research.

\section{References}

CivCO, D.L., 1989, Topographic normalization of Landsat thematic mapper digital imagery. Photogrammetric Engineering and Remote Sensing, 55, pp. 1303-1309.

Holben, B.N. and Justice, C.O., 1980, The topographic effect on spectral response from nadir-pointing sensors. Photogrammetric Engineering and Remote Sensing, 46, pp. $1191-1200$.

LAw, K.H., 2005, Correction of terrain effects on satellite image radiance. M. Phil dissertation, Department of Land Surveying and GeoInformatics, The Hong Kong Polytechnic University.

Meyer, P., Itten, K., Kellenberger, T., Sandmeier, S. and Sandmeier, R., 1993, Radiometric corrections of topographically induced effects on Landsat TM data in an alpine environment. ISPRS Journal of Photogrammetry and Remote Sensing, 48, pp. $17-28$.

MinnAert, M., 1941, The reciprocity principle in lunar photometry. Astrophysics Journal, 93, pp. $403-410$.

ShePherd, J.D. and Dymond, J.R., 2003, Correcting satellite imagery for the variance of reflectance and illumination with topography. International Journal of Remote Sensing, 24, pp. 3503-3514.

Smith, J.A., Lin, T.L. and Ranson, K.J., 1980, The Lambertian assumption and Landsat data. Photogrammetric Engineering and Remote Sensing, 46, pp. 1183-1189.

Teillet, P.M., Guindon, B. and Goodenough, D.G., 1982, On the slope-aspect correction of multispectral scanner data. Canadian Journal of Remote Sensing, 8, pp. 1537-1540. 